\title{
Teleportation and spin squeezing utilizing multimode entanglement of light with atoms
}

\author{
K. Hammerer ${ }^{1}$, E.S. Polzik ${ }^{2,3}$, J.I. Cirac ${ }^{1}$ \\ ${ }^{1}$ Max-Planck-Institut für Quantenoptik, Hans-Kopfermann-Strasse, D-85748 Garching, Germany \\ ${ }^{2}$ QUANTOP, Danish Research Foundation Center for Quantum Optics, DK 2100 Copenhagen, Denmark \\ ${ }^{3}$ Niels Bohr Institute, DK 2100 Copenhagen, Denmark
}

\begin{abstract}
We present a protocol for the teleportation of the quantum state of a pulse of light onto the collective spin state of an atomic ensemble. The entangled state of light and atoms employed as a resource in this protocol is created by probing the collective atomic spin, Larmor precessing in an external magnetic field, off resonantly with a coherent pulse of light. We take here for the first time full account of the effects of Larmor precession and show that it gives rise to a qualitatively new type of multimode entangled state of light and atoms. The protocol is shown to be robust against the dominating sources of noise and can be implemented with an atomic ensemble at room temperature interacting with free space light. We also provide a scheme to perform the readout of the Larmor precessing spin state enabling the verification of successful teleportation as well as the creation of spin squeezing.
\end{abstract}

PACS numbers: 03.67.Mn, 32.80.Qk

\section{INTRODUCTION}

Quantum teleportation - the disembodied transport of quantum states - has been demonstrated so far in several seminal experiments dealing with purely photonic 1] or atomic 2] systems. Here we propose a protocol for the teleportation of a coherent state carried initially by a pulse of light onto the collective spin state of $\sim 10^{11}$ atoms. This protocol - just as the recently demonstrated direct transfer of a quantum state of light onto atoms [3] - is particularly relevant for long distance entanglement distribution, a key resource in quantum communication networks [4].

Our scheme can be implemented with just coherent light and room-temperature atoms in a single vapor cell placed in a homogeneous magnetic field. Existing protocols in Quantum Information (QI) with continuous variables of atomic ensembles and light [4] are commonly designed for setups where no external magnetic field is applied such that the interaction of light with atoms meets the Quantum non-demolition (QND) criteria [5, 6]. In contrast, in all experiments dealing with vapor cells at room-temperature [3, 7] it is, for technical reasons, absolutely essential to employ magnetic fields. In experiments [3, 7] two cells with counter-rotating atomic spins were used to comply with both, the need for an external magnetic field and the one for an interaction of QND character. So far it was believed to be impossible to use a single cell in a magnetic field to implement QI protocols, since in this case - due to the Larmor precession - scattered light simultaneously reads out two non-commuting spin components such that the interaction is not of QND type.

In this paper we do not only show that it is well possible to make use of the quantum state of light and atoms created in this setup but we demonstrate that - for the purpose of teleportation [8, [9] - it is in fact better to do so. As compared to the state resulting from the common QND interaction the application of an external magnetic field enhances the creation of correlations between atoms and light, generating more and qualitatively new, multimode type of entanglement. The results of the paper can be summarized as follows:

(i) Larmor precession in an external magnetic field enhances the creation of entanglement when a collective atomic spin is probed with off-resonant light. The resulting entanglement involves multiple modes and is stronger as compared to what can be achieved in a comparable QND interaction.

(ii) This type of entangled state can be used as a resource in a teleportation protocol, which is a simple generalization of the standard protocol [8, 9$]$ based on Einstein-Podolsky-Rosen (EPR) type of entanglement. For the experimentally accessible parameter regime the teleportation fidelity is close to optimal. The protocol is robust against imperfections and can be implemented with state of the art technique.

(iii) Homodyne detection of appropriate scattering modes of light leaves the atomic state in a spin squeezed state. The squeezing can be the same as attained from a comparable QND measurement of the atomic spin 10, 11]. The same scheme can be used for atomic state read-out of the Larmor precessing spin, necessary to verify successful teleportation.

We would like to note that it was shown recently in 12] that the effect of a magnetic field can enhance the capacity of a quantum memory in the setup of two cells. Teleportation in the setup of a single cell without magnetic field was addressed in [13].

The paper is organized as follows: The three points above are presented in sections [II III and IV in this order. Some of the details in the calculations of sections III] and IV] are moved to appendices B and [C] 


\section{INTERACTION}

We consider an ensemble of $N_{a t}$ Alkali atoms with total ground state angular momentum $F$, placed in a constant magnetic field causing a Zeeman splitting of $\hbar \Omega$ and initially prepared in a fully polarized state along $x$. The collective spin of the ensemble is then probed by an off resonant pulse which propagates along $z$ and is linearly polarized along $x$. Thorough descriptions of this interaction and the final state of light and atoms after the scattering can be found in 14, 15, 16, 17, 18] and especially in 19, 20, 21, 22] for the specific system we have in mind. We derive the final state here with a special focus on the effects of Larmor precession and light propagation in order to identify the light modes which are actually populated in the scattering process.

In appendix $\mathrm{A}$ we show that the interaction is adequately described by a Hamiltonian

$$
\begin{aligned}
H & =H_{a t}+H_{l i}+V, \\
H_{a t} & =\frac{\hbar \Omega}{2}\left(X^{2}+P^{2}\right), \\
V & =\frac{\hbar \kappa}{\sqrt{T}} P p(0)
\end{aligned}
$$

and where $H_{l i}$ is the Hamiltonian for the free radiation field. The canonical conjugate variables $X, P$ describe in the Holstein-Primakoff approximation [23] transverse components of the collective angular momentum in $y$ and $z$ direction respectively. They satisfy $[X, P]=i$ and have zero mean and a normalized variance $\Delta X^{2}=\Delta P^{2}=1 / 2$ for the initial coherent spin state. In analogy to light field quadratures we will denote the normalized transverse spin components $X, P$ also as spin quadratures. For the light field only its linearly polarized component along $y$ is relevant and is described in terms of quadratures of spatially localized modes [24, 25], $x(z), p(z)$, which obey $\left[x(z), p\left(z^{\prime}\right)\right]=i c \delta\left(z-z^{\prime}\right)$. Before the interaction process, this polarization component is in vacuum such that initially $\langle x(z)\rangle=\langle p(z)\rangle=0$ and $\left\langle x(z) x\left(z^{\prime}\right)\right\rangle=\left\langle p(z) p\left(z^{\prime}\right)\right\rangle=c \delta\left(z-z^{\prime}\right) / 2$. The dimensionless coupling constant is given by $\kappa=\sqrt{N_{p h} N_{a t} F} a_{1} \sigma \Gamma / 2 A \Delta$ where $N_{p h}$ is the overall number of photons in the pulse, $a_{1}$ is a constant characterizing the ground state's vector polarizability, $\sigma$ is the scattering cross section, $\Gamma$ the decay rate, $\Delta$ the detuning and $A$ the effective beam cross section.

Changing to a rotating frame with respect to $H_{a t}$ by defining $X_{I}(t)=\exp \left(-i H_{a t} t\right) X \exp \left(i H_{a t} t\right)$ and evaluating the Heisenberg equations for these operators yields the following Maxwell-Bloch equations

$$
\begin{aligned}
\partial_{t} X_{I}(t) & =\frac{\kappa}{\sqrt{T}} \cos (\Omega t) p(0, t), \\
\partial_{t} P_{I}(t) & =\frac{\kappa}{\sqrt{T}} \sin (\Omega t) p(0, t), \\
\left(\partial_{t}+c \partial_{z}\right) x(z, t) & =\frac{\kappa c}{\sqrt{T}}\left[\cos (\Omega t) P_{I}(t)-\sin (\Omega t) X_{I}(t)\right] \delta(z), \\
\left(\partial_{t}+c \partial_{z}\right) p(z, t) & =0
\end{aligned}
$$

and a sine component $p_{\mathrm{s}}^{\text {in }}, x_{\mathrm{s}}^{\text {in }}$ with $\cos (\Omega \tau)$ replaced by $\sin (\Omega \tau)$. In frequency space these modes consist of spectral components at sidebands $\omega_{c} \pm \Omega$ and are closely related to the sideband modulation modes introduced in

where $\partial_{t(z)}$ denotes the partial derivative with respect to $t(z)$. These equations have a clear interpretation. Light noise coming from the field in quadrature with the classical probe piles up in both, the $X$ and $P$ spin quadrature, but it alternately affects only one or the other, changing with a period of $1 / \Omega$. Conversely atomic noise adds to the in phase field quadrature only and the signal comes alternately from the $X$ and $P$ spin quadrature. The out of phase field quadrature is conserved in the interaction.

To solve this set of coupled equations it is convenient to introduce a new position variable, $\xi=c t-z$, to eliminate the $z$ dependence. New light quadratures defined by $\bar{x}(\xi, t)=x(c t-\xi, t), \bar{p}(\xi, t)=p(c t-\xi, t)$ also have a simple interpretation: $\xi$ labels the slices of the pulse moving in and out of the ensemble one after the other, starting with $\xi=0$ and terminating at $\xi=c T$. The Maxwell equations now read

$$
\begin{aligned}
& \partial_{t} \bar{p}(\xi, t)=0, \\
& \partial_{t} \bar{x}(\xi, t)=\frac{\kappa c}{\sqrt{T}}\left[\cos (\Omega t) P_{I}(t)-\sin (\Omega t) X_{I}(t)\right] \delta(c t-\xi) .
\end{aligned}
$$

The solutions to equations $(2 \mathrm{a}, 2 \mathrm{~b}, 2 \mathrm{c})$ are

$$
\begin{aligned}
X_{I}(t) & =X_{I}(0)+\frac{\kappa}{\sqrt{T}} \int_{0}^{t} \mathrm{~d} \tau \cos (\Omega \tau) \bar{p}(c \tau, 0), \\
P_{I}(t) & =P_{I}(0)+\frac{\kappa}{\sqrt{T}} \int_{0}^{t} \mathrm{~d} \tau \sin (\Omega \tau) \bar{p}(c \tau, 0), \\
\bar{p}(\xi, t) & =\bar{p}(\xi, 0)
\end{aligned}
$$

and the formal solution to $(2 \mathrm{~d})$ is

$$
\begin{aligned}
\bar{x}(\xi, t) & =\bar{x}(\xi, 0)+ \\
& +\frac{\kappa}{\sqrt{T}}\left[\cos (\Omega \xi / c) P_{I}(\xi / c)-\sin (\Omega \xi / c) X_{I}(\xi / c)\right] .
\end{aligned}
$$

As mentioned before, both atomic spin quadratures are affected by light but, as is evident from the solutions for $X(t), P(t)$, they receive contributions from different and, in fact, orthogonal projections of the out-of-phase field. As we will show in the following, the corresponding projections of the in-phase field carry in turn the signal of atomic quadratures after the interaction. It is therefore convenient to explicitly introduce operators for these modes [21]. We define a cosine component before the interaction

$$
\begin{aligned}
& p_{\mathrm{c}}^{\text {in }}=\sqrt{\frac{2}{T}} \int_{0}^{T} \mathrm{~d} \tau \cos (\Omega \tau) \bar{p}(c \tau, 0), \\
& x_{\mathrm{c}}^{\text {in }}=\sqrt{\frac{2}{T}} \int_{0}^{T} \mathrm{~d} \tau \cos (\Omega \tau) \bar{x}(c \tau, 0)
\end{aligned}
$$


[26] for the description of two photon processes. It is easily checked that these modes are asymptotically canonical, $\left[x_{\mathrm{c}}^{\mathrm{in}}, p_{\mathrm{c}}^{\mathrm{in}}\right]=\left[x_{\mathrm{s}}^{\mathrm{in}}, p_{\mathrm{s}}^{\mathrm{in}}\right]=i\left[1+\mathcal{O}\left(n_{0}^{-1}\right)\right] \simeq i$, and independent, $\left[x_{\mathrm{c}}^{\mathrm{in}}, p_{\mathrm{s}}^{\text {in }}\right]=\mathcal{O}\left(n_{0}^{-1}\right) \simeq 0$, if we assume $n_{0} \gg 1$ for $n_{0}=\Omega T$, the pulse length measured in periods of Larmor precession.

In terms of these modes the atomic state after the interaction $X^{\text {out }}=X_{I}(T), P^{\text {out }}=P_{I}(T)$ is given by

$$
X^{\text {out }}=X^{\text {in }}+\frac{\kappa}{\sqrt{2}} p_{\mathrm{c}}^{\text {in }}, \quad P^{\text {out }}=P^{\text {in }}+\frac{\kappa}{\sqrt{2}} p_{\mathrm{s}}^{\text {in }} .
$$

The final state of cosine (sine) modes is described by $x_{\mathrm{c}(\mathrm{s})}^{\text {out }}, p_{\mathrm{c}(\mathrm{s})}^{\text {out }}$, defined by equations (4) with $\bar{x}(c \tau, 0), \bar{p}(c \tau, 0)$ replaced by $\bar{x}(c \tau, T), \bar{p}(c \tau, T)$ respectively. Since the out-of-phase field is conserved we have trivially

$$
p_{\mathrm{c}}^{\text {out }}=p_{\mathrm{c}}^{\text {in }}, \quad p_{\mathrm{s}}^{\text {out }}=p_{\mathrm{s}}^{\text {in }} .
$$

Deriving the corresponding expressions for the cosine and sine components of the field in phase, $x_{\mathrm{c}}^{\text {out }}, x_{\mathrm{s}}^{\text {out }}$, raises some difficulties connected to the back action of light onto itself. This effect can be understood by noting that a slice $\xi$ of the pulse receives a signal of atoms at a time $\xi / c$ [see equation (3d)] which, regarding equations Ba 3b), in turn carry already the integrated signal of all slices up to $\xi$. Thus, mediated by the atoms, light acts back on itself. The technicalities in the treatment of this effect are given in appendix $\mathrm{B}$ where we identify relevant "back action modes", $x_{\mathrm{c}, 1}, p_{\mathrm{c}, 1}, x_{\mathrm{s}, 1}, p_{\mathrm{s}, 1}$, in terms of which one can express

$$
\begin{aligned}
& x_{\mathrm{c}}^{\text {out }}=x_{\mathrm{c}}^{\text {in }}+\frac{\kappa}{\sqrt{2}} P^{\text {in }}+\left(\frac{\kappa}{2}\right)^{2} p_{\mathrm{s}}^{\text {in }}+\frac{1}{\sqrt{3}}\left(\frac{\kappa}{2}\right)^{2} p_{\mathrm{s}, 1}^{\text {in }}, \\
& x_{\mathrm{s}}^{\text {out }}=x_{\mathrm{s}}^{\text {in }}-\frac{\kappa}{\sqrt{2}} X^{\text {in }}-\left(\frac{\kappa}{2}\right)^{2} p_{\mathrm{c}}^{\text {in }}-\frac{1}{\sqrt{3}}\left(\frac{\kappa}{2}\right)^{2} p_{\mathrm{c}, 1}^{\text {in }} .
\end{aligned}
$$

The last two terms in both lines represent the effect of back action, part of which involves the already defined cosine and sine components of the field in quadrature. The remaining part is subsumed in the back action modes which are again canonical and independent from all other modes.

Equations (5) describe the final state of atoms and the relevant part of scattered light after the pulse has passed the atomic ensemble and are the central result of this section. Treating the last terms in equations (5c5d) as noise terms, it is readily checked by means of the separability criteria in [27] that this state is fully inseparable, i.e. it is inseparable with respect to all splittings between the three modes. For the following teleportation protocol the relevant entanglement is the one between atoms and the two light modes. Figure 1 shows the von Neumann entropy $E_{\mathrm{vN}}$ of the reduced state of atoms in its dependence on the coupling strength $\kappa$ and in comparison with the entanglement created without magnetic field in a pure QND interaction of atoms and light. The amount of entanglement is significantly enhanced.

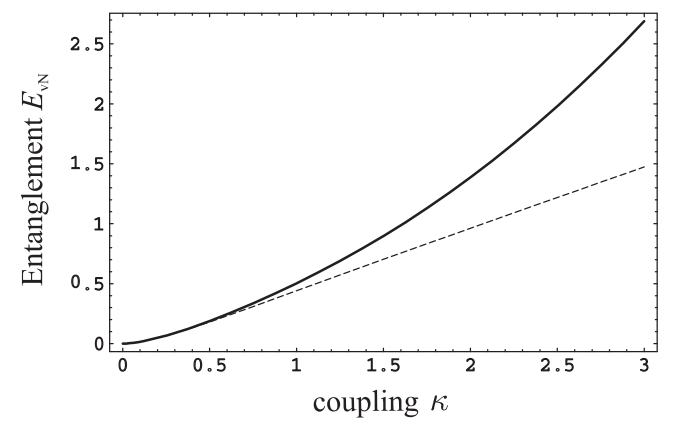

FIG. 1: Von Neumann Entropy of the reduced state of atoms versus coupling strength kappa for the state of equation [5] (full line) and for the state generated without magnetic field in a pure QND interaction (dashed line) with the same coupling strength. Application of a magnetic field significantly enhances the amount of light-atom entanglement.

\section{TELEPORTATION OF LIGHT ONTO ATOMS}

In this section we will show how the multimode entanglement between light and atoms generated in the scattering process can be employed in a teleportation protocol which is a simple generalization of the standard protocol for continuous variable teleportation using EPR-type entangled states [8, 9]. We first present the protocol and evaluate its fidelity and then analyze its performance under realistic experimental conditions.

\section{A. Basic protocol}

Figure 2 depicts the basic scheme which, as usually, consists of a Bell measurement and a feedback operation.

Input The coherent state to be teleported is encoded in a pulse which is linearly polarized orthogonal to the classical driving pulse and whose carrier frequency lies at the upper sideband, i.e. at $\omega_{c}+\Omega$. The pulse envelope has to match the one of the classical pulse. As is shown in appendix B canonical operators $y, q$ with $[y, q]=i$ describing this mode can conveniently be expressed in terms of cosine and sine modulation modes, analogous to equations (4), defined with respect to the carrier frequency. One finds

$$
y=\frac{1}{\sqrt{2}}\left(y_{\mathrm{s}}+q_{\mathrm{c}}\right), \quad q=-\frac{1}{\sqrt{2}}\left(y_{\mathrm{c}}-q_{\mathrm{s}}\right) .
$$

A coherent input amounts to having initially $\Delta y^{2}=\Delta q^{2}=1 / 2$ and an amplitude $\langle y\rangle,\langle q\rangle$ with mean photon number $n_{p h}=\left(\langle y\rangle^{2}+\langle q\rangle^{2}\right) / 2$.

Bell measurement This input is combined at a beam splitter with the classical pulse and the scattered light. At the ports of the beam splitter Stokes vector components $S_{y}$ and $S_{z}$ are measured by means of standard polarization measurements. Given the classical pulse in $x$ 


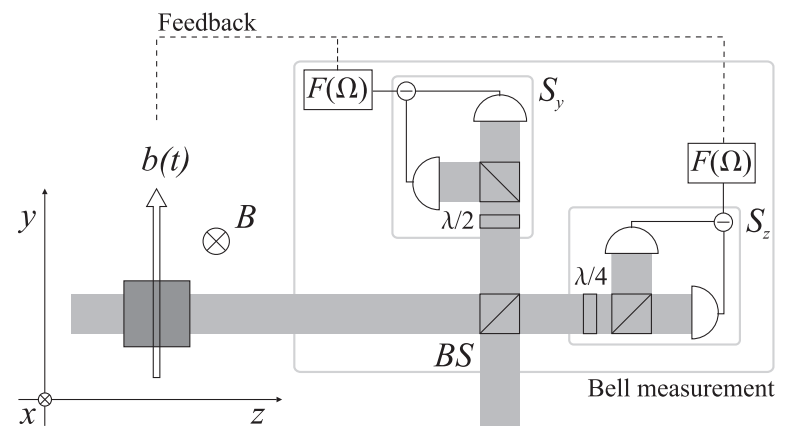

FIG. 2: Scheme for teleportation of light onto atoms: As described in section II a classical pulse (linearly polarized along $x$ ) propagating along the positive $z$ direction is scattered off an atomic ensemble contained in a glass cell and placed in a constant magnetic field $B$ along $x$. Classical pulse and scattered light (linearly polarized along $y$ ) are overlapped with a with a coherent pulse (linearly polarized along $z$ ) at beam splitter $B S$. By means of standard polarization measurements Stokes vector components $S_{y}$ and $S_{z}$ are measured at one and the other port respectively, realizing the Bell measurement. The Fourier components at Larmor frequency $\Omega$ of the corresponding photocurrents determine the amount of conditional displacement of the atomic spin which can be achieved by applying a properly timed transverse magnetic field $b(t)$. See section IIIA for details.

polarization this amounts to a homodyne detection of in- and out-of-phase fields of the orthogonal polarization component. The resulting photocurrents are numerically demodulated to extract the relevant sine and cosine components at the Larmor frequency [20]. Thus one effectively measures the commuting observables

$$
\begin{array}{ll}
\tilde{x}_{\mathrm{c}}=\frac{1}{\sqrt{2}}\left(x_{\mathrm{c}}^{\text {out }}+y_{\mathrm{c}}\right), & \tilde{x}_{\mathrm{s}}=\frac{1}{\sqrt{2}}\left(x_{\mathrm{s}}^{\text {out }}+y_{\mathrm{s}}\right), \\
\tilde{q}_{\mathrm{c}}=\frac{1}{\sqrt{2}}\left(p_{\mathrm{c}}^{\text {out }}-q_{\mathrm{c}}\right), & \tilde{q}_{\mathrm{s}}=\frac{1}{\sqrt{2}}\left(p_{\mathrm{s}}^{\text {out }}-q_{\mathrm{s}}\right) .
\end{array}
$$

Let the respective measurement results be given by $\tilde{X}_{\mathrm{c}}, \tilde{X}_{\mathrm{s}}, \tilde{Q}_{\mathrm{c}}$ and $\tilde{Q}_{\mathrm{s}}$.

Feedback Conditioned on these results the atomic state is then displaced by an amount $\tilde{X}_{\mathrm{s}}-\tilde{Q}_{\mathrm{c}}$ in $X$ and $-\tilde{X}_{\mathrm{c}}-\tilde{Q}_{\mathrm{s}}$ in $P$. This can be achieved by means of two fast radio-frequency magnetic pulses separated by a quarter of a Larmor period. In the ensemble average the final state of atoms is simply given by

$$
X^{\text {fin }}=X^{\text {out }}+\tilde{x}_{\mathrm{s}}-\tilde{q}_{\mathrm{c}}, \quad P^{\text {fin }}=P^{\text {out }}-\tilde{x}_{\mathrm{c}}-\tilde{q}_{\mathrm{s}} .
$$

This description of feedback is justified rigorously in appendix [ Relating these expressions to input operators, we find by means of equations (5), (6) and (7)

$$
\begin{aligned}
X^{\mathrm{fin}}= & \left(1-\frac{\kappa}{2}\right) X^{\mathrm{in}}-\frac{1}{\sqrt{2}}\left(1-\frac{\kappa}{2}\right)^{2} p_{\mathrm{c}}^{\text {in }} \\
& +\frac{1}{\sqrt{2}} x_{\mathrm{s}}^{\mathrm{in}}-\frac{1}{\sqrt{6}}\left(\frac{\kappa}{2}\right)^{2} p_{\mathrm{c}, 1}^{\text {in }}+y, \\
P^{\mathrm{fin}}= & \left(1-\frac{\kappa}{2}\right) P^{\mathrm{in}}-\frac{1}{\sqrt{2}}\left(1-\frac{\kappa}{2}\right)^{2} p_{\mathrm{s}}^{\text {in }} \\
& -\frac{1}{\sqrt{2}} x_{\mathrm{c}}^{\mathrm{in}}-\frac{1}{\sqrt{6}}\left(\frac{\kappa}{2}\right)^{2} p_{\mathrm{s}, 1}^{\text {in }}+q .
\end{aligned}
$$

This is the main result of this section.

Teleportation fidelity Taking the mean of equations (9) with respect to the initial state all contributions due to input operators and back action modes vanish such that $\left\langle X^{\text {fin }}\right\rangle=\langle y\rangle$ and $\left\langle P^{\text {fin }}\right\rangle=\langle q\rangle$. Thus, the amplitude of the coherent input light pulse is mapped on atomic spin quadratures as desired. In order to prove faithful teleportation also the variances have to be conserved. It is evident from (9) that the final atomic spin variances will be increased as compared to the coherent input. These additional terms describe unwanted excess noise and have to be minimized by a proper choice of the coupling $\kappa$. As a figure of merit for the teleportation protocol we use the fidelity, i.e. squared overlap, of input and final state. Given that the means are transmitted correctly the fidelity is found to be $F=$ $2\left[\left(1+2\left(\Delta X^{\text {fin }}\right)^{2}\right)\left(1+2\left(\Delta P^{\text {fin }}\right)^{2}\right)\right]^{-1 / 2}$. The variances of the final spin quadratures are readily calculated taking into account that all modes involved are independent and have initially a normalized variance of $1 / 2$. In this way a theoretical limit on the achievable fidelity can be derived depending solely on the coupling strength $\kappa$. In figure 3 we take advantage of the fact that the amount of entanglement between light and atoms is a monotonously increasing function of $\kappa$ such that we can plot the fidelity versus the entanglement. This has the advantage that we can compare the performance of our teleportation protocol with the canonical one [8, 9] which uses a two-mode squeezed state of the same entanglement as a resource and therefore maximizes the teleportation fidelity for the given amount of entanglement. No physical state can achieve a higher fidelity with the same entanglement. This follows from the results of [30] where it was shown that two-mode squeezed states minimize the EPR variance (and therefore maximize the teleportation fidelity) for given entanglement. The theoretical fidelity achievable in our protocol is maximized for $\kappa \simeq 1.64$ corresponding to $F \simeq .77$. But also for experimentally more feasible values of $\kappa \simeq 1$ can the fidelity well exceed the classical limit 28, 29] of $1 / 2$ and, moreover, comparison with the values achievable with a two-mode squeezed state shows that our protocol is close to optimal. 


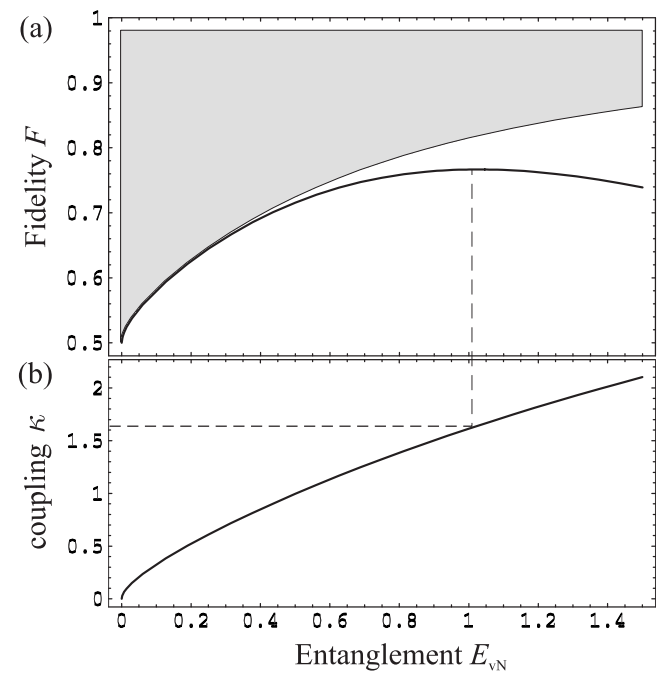

FIG. 3: (a) Theoretical limit on the achievable fidelity $F$ versus entanglement between atoms and light measured by the von Neumann entropy $E_{\mathrm{vN}}$ of the reduced state of atoms. The grey area is unphysical. For moderate amounts of entanglement our protocol is close to optimal. (b) Coupling strength $\kappa$ versus entanglement. The dashed lines indicate the maximal fidelity of $F=.77$ which is achieved for $\kappa=1.64$.

\section{B. Noise effects and Gaussian distributed input}

Under realistic conditions the teleportation fidelity will be degraded by noise effects like decoherence of the atomic spin state, light absorption and reflection losses and also because the coupling constant $\kappa$ is experimentally limited to values $\kappa \simeq 1$. On the other hand the classical fidelity bound to be beaten will be somewhat higher than $1 / 2$ since the coherent input states will necessarily be drawn according to a distribution with a finite width in the mean photon number $\bar{n}$. In this section we analyze the efficiency of the teleportation protocol under these conditions and show that it is still possible to surpass any classical strategy for the transmission and storage of coherent states of light [28, 29].

During the interaction atomic polarization decays due to spontaneous emission and collisional relaxation. Including a transverse decay the final state of atoms is given by

$$
\begin{aligned}
& X^{\text {out }}=\sqrt{1-\beta}\left(X^{\text {in }}+\frac{\kappa}{\sqrt{2}} p_{\mathrm{c}}^{\text {in }}\right)+\sqrt{\beta} f_{X}, \\
& P^{\text {out }}=\sqrt{1-\beta}\left(P^{\text {in }}+\frac{\kappa}{\sqrt{2}} p_{\mathrm{s}}^{\text {in }}\right)+\sqrt{\beta} f_{P} .
\end{aligned}
$$

as follows from the discussion in appendix $\beta$ is the atomic decay parameter and $f_{X}, f_{P}$ are Langevin noise operators with zero mean. Their variance is experimentally found to be close to the value corresponding to a coherent state such that $\left\langle f_{X}^{2}\right\rangle=\left\langle f_{P}^{2}\right\rangle=1 / 2$.

Light absorption and reflection losses can be taken into account in the same way as finite detection efficiency. For example the statistics of measurement outcome $\tilde{X}_{\mathrm{s}}$ will not stem from the signal mode $\tilde{x}_{\mathrm{s}}$ alone but rather from the noisy mode $\sqrt{1-\epsilon} \tilde{x}_{\mathrm{s}}+\sqrt{\epsilon} f_{x, \mathrm{~s}}$ where $\epsilon$ is the photon loss parameter and $f_{x, s}$ is a Langevin noise operator of zero mean and variance $\left\langle f_{x, s}^{2}\right\rangle=1 / 2$. Analogous expressions have to be used for the measurements of $\tilde{x}_{\mathrm{c}}, \tilde{q}_{\mathrm{s}}$ and $\tilde{q}_{\mathrm{c}}$ which will be adulterated by Langevin terms $f_{x, \mathrm{c}}, f_{q, \mathrm{~s}}$ and $f_{q, \mathrm{c}}$ respectively. In principle each of the measurement outcomes can be fed back with an independently chosen gain but for symmetry reasons it is enough to distinguish gain coefficients $g_{x}, g_{q}$ for the measurement outcomes of sine and cosine components of $x$ and $q$ respectively. Including photon loss, finite gain and atomic decay, as given in (10), equations (8), describing the final state of atoms after the feed back operation, generalize to

$$
\begin{aligned}
X^{\text {fin }=} & \sqrt{1-\beta} X^{\text {out }}+\sqrt{\beta} f_{X} \\
& +g_{x}\left(\sqrt{1-\epsilon} \tilde{x}_{\mathrm{s}}+\sqrt{\epsilon} f_{x, \mathrm{~s}}\right) \\
& -g_{q}\left(\sqrt{1-\epsilon} \tilde{q}_{\mathrm{c}}+\sqrt{\epsilon} f_{q, \mathrm{c}}\right), \\
P^{\text {fin }}= & \sqrt{1-\beta} P^{\text {out }}+\sqrt{\beta} f_{P} \\
& -g_{x}\left(\sqrt{1-\epsilon} \tilde{x}_{\mathrm{c}}+\sqrt{\epsilon} f_{x, \mathrm{c}}\right) \\
& -g_{q}\left(\sqrt{1-\epsilon} \tilde{q}_{\mathrm{s}}+\sqrt{\epsilon} f_{q, \mathrm{~s}}\right) .
\end{aligned}
$$

For non unit gains a given coherent amplitude $(\langle y\rangle,\langle q\rangle)$ will not be perfectly teleported onto atoms and the corresponding fidelity will be degraded by this mismatch according to

$$
\begin{aligned}
F(\langle y\rangle,\langle q\rangle)= & \frac{2}{\sqrt{\left[1+2\left(\Delta X^{\text {fin }}\right)^{2}\right]\left[1+2\left(\Delta P^{\text {fin }}\right)^{2}\right]}} \\
& \cdot \exp \left[-\frac{\left(\langle y\rangle-\left\langle X^{\text {fin }}\right\rangle\right)^{2}}{1+2\left(\Delta X^{\text {fin }}\right)^{2}}-\frac{\left(\langle q\rangle-\left\langle P^{\text {fin }}\right\rangle\right)^{2}}{1+2\left(\Delta P^{\text {fin }}\right)^{2}}\right] .
\end{aligned}
$$

If the input amplitudes are drawn according to a Gaussian distribution $p(\langle y\rangle,\langle q\rangle)=\exp \left[-\left(\langle y\rangle^{2}+\langle q\rangle^{2}\right) / 2 \bar{n}\right] / 2 \pi \bar{n} \quad$ with mean photon number $\bar{n}$ the average fidelity [with respect to $(\langle y\rangle,\langle q\rangle)]$ is readily calculated. The exact expression in terms of initial operators can then be derived by means of equations (5), (6), (7) and (11) but is not particularly enlightening. In figure 4 we plot the average fidelity, optimized with respect to gains $g_{x}, g_{q}$, in its dependence on the atomic decay $\beta$ for various values of photon loss $\epsilon$. We assume a realistic value $\kappa=0.96$ for the coupling constant and a mean number of photons $\bar{n}=4$ for the distribution of the coherent input. For feasible values of $\beta, \epsilon \precsim 0.2$ the average fidelity is still well above the classical bound on the fidelity [28, 29]. This proves that the proposed protocol is robust against the dominating noise effects in this system.

The experimental feasibility of the proposal is illustrated with the following example. Consider a sample of $N_{a t}=10^{12}$ Cesium atoms in a glass cell placed in a constant magnetic field along the $x$-direction causing a Zeeman splitting of $\Omega=350 \mathrm{kHz}$ in the $F=4$ ground state multiplet. The atoms are pumped into $m_{F}=4$ and probed on the $\mathrm{D}_{2}\left(F=4 \rightarrow F^{\prime}=3,4,5\right)$ 


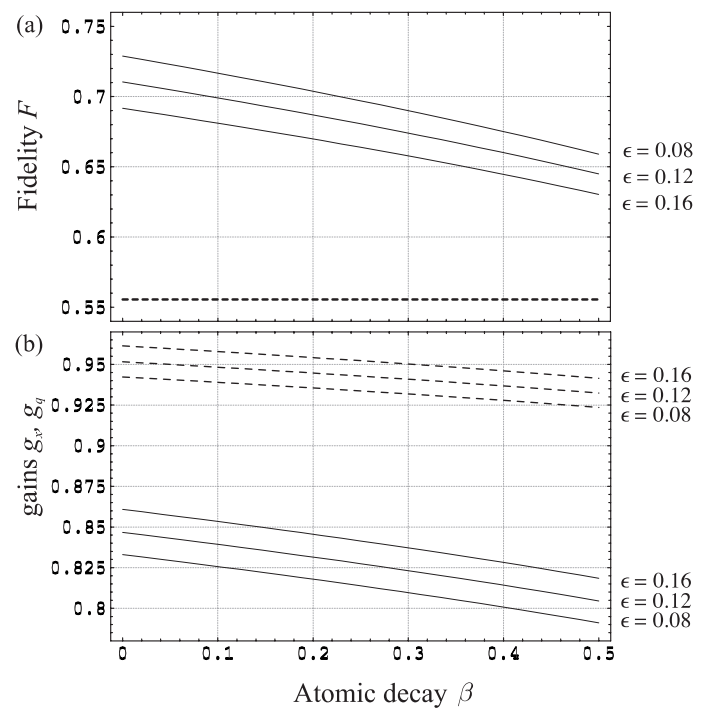

FIG. 4: (a) Average fidelity achievable in the presence of atomic decay $\beta$, reflection and light absorption losses $\epsilon=8 \%, 12 \%, 16 \%$, coupling $\kappa=0.96$ and Gaussian distributed input states with mean photon number $\bar{n}=4$. The fidelity benchmark is in this case 5/9 (dashed line). (b) Respective optimal values for gains $g_{x}$ (solid lines) and $g_{q}$ (dashed lines).

transition. The classical pulse contains an overall number of $N_{p h}=2.510^{13}$ photons, is detuned to the blue by $\Delta=1 \mathrm{GHz}$, has a duration $T=1 \mathrm{~ms}$ and can have an effective cross section of $A \simeq 6 \mathrm{~cm}^{2}$ due to thermal motion of atoms. Under these conditions the tensor polarizability can be neglected $\left(\Delta / \omega_{\mathrm{hfs}} \simeq 10^{-1}\right)$. Also $n_{0}=\Omega T=350$ justifies the use of independent scattering modes. The coupling $\kappa \simeq 1$ and the depumping of ground state population $\eta \simeq 10^{-1}$ as desired.

\section{SPIN SQUEEZING AND STATE READ-OUT}

In this section we present a scheme for reading out either of the atomic spin components $X, P$ by means of a probe pulse interacting with the atoms in the one way as described in section III The proposed scheme allows one, on the one hand, to verify successful receipt of the coherent input subsequent to the teleportation protocol of section III and, on the other hand, enables to generate spin squeezing if it is performed on a coherent spin state.

It is well known [15, 31] and was demonstrated experimentally [10, 11] that the pure interaction $V$, as given in equation (11), can be used to perform a QND measurement of either of the transverse spin components. At first sight this seems not to be an option in the scenario under consideration since the local term $H_{a t}$, accounting for Larmor precession, commutes with neither of the spin quadratures such that the total Hamiltonian does not satisfy the QND criteria [5, 6]. As we have shown in section II Larmor precession has two effects: Scattered light is correlated with both transverse components and suffers from back action mediated by the atoms. Thus, in order to read out a single spin component one has to overcome both disturbing effects.

Our claim is that this can be achieved by a simultaneous measurement of $x_{\mathrm{c}}^{\text {out }}, p_{\mathrm{s}}^{\text {out }}, p_{\mathrm{s}, 1}^{\text {out }}$ or $x_{\mathrm{s}}^{\text {out }}, p_{\mathrm{c}}^{\text {out }}, p_{\mathrm{c}, 1}^{\text {out }}$ if, respectively, $X$ or $P$ is to be measured. In the following we consider in particular the former case but everything will hold with appropriate replacements also for a measurement of $P$.

As shown in figure 5 the set of observables $x_{\mathrm{c}}^{\text {out }}, p_{\mathrm{s}}^{\text {out }}, p_{\mathrm{s}, 1}^{\text {out }}$ can be measured simultaneously by a measurement of Stokes component $S_{y}$ after a $\pi / 2$ rotation is performed selectively on the sine component of the scattered light. The cosine component of the corresponding photocurrent will give an estimate of $x_{\mathrm{c}}^{\text {out }}$ and the sine component of $p_{\mathrm{s}}^{\text {out }}$. Multiplying the photocurrent's sine component by the linear function defining the back action mode, equation (B1), will give in addition an estimate of $p_{\mathrm{s}, 1}^{\text {out }}$. Note that the field out of phase is conserved in the interaction such that

$$
p_{\mathrm{s}, 1}^{\text {out }}=p_{\mathrm{s}, 1}^{\text {in }}, \quad p_{\mathrm{c}, 1}^{\text {out }}=p_{\mathrm{c}, 1}^{\text {in }},
$$

i.e. the results will have shot noise limited variance. It is then evident from equation (5c) that the respective photocurrents together with an a priori knowledge of $\kappa$ are sufficient to estimate the mean $\langle X\rangle$.

The conditional variances after the indicated measurements are

$$
\begin{aligned}
\left.\Delta X^{2}\right|_{\left\{x_{\mathrm{c}}^{\text {out }}, p_{\mathrm{s}}^{\text {out }}, p_{\mathrm{s}, 1}^{\text {out }}\right\}} & =\left(\Delta X^{\mathrm{in}}\right)^{2} \frac{2}{2+\kappa^{2}}, \\
\left.\Delta P^{2}\right|_{\left\{x_{\mathrm{c}}^{\text {out }}, p_{\mathrm{s}}^{\text {out }}, p_{\mathrm{s}, 1}^{\text {out }}\right\}} & =\left(\Delta P^{\mathrm{in}}\right)^{2} \frac{2+\kappa^{2}}{2},
\end{aligned}
$$

corresponding to a pure state. Obviously the variance in $X$ is squeezed by a factor $\left(1+\kappa^{2} / 2\right)^{-1}$. Note that the squeezing achieved in a QND measurement without magnetic field but otherwise identical parameters is given by $\left(1+\kappa^{2}\right)^{-1}$. From this we conclude that the quality of the estimate for $\langle X\rangle$, as measured f.e. by input-output coefficients known from the theory of QND measurements [5, 6], can be the same as in the case without Larmor precession albeit only for a higher coupling $\kappa$.

Equations (13) are conveniently derived by means of the formalism of correlation matrices 32]. For the operator valued vector $\vec{R}=(X, P$, $\left.x_{\mathrm{c}}, p_{\mathrm{c}}, x_{\mathrm{s}}, p_{\mathrm{s}}, x_{\mathrm{c}, 1}, p_{\mathrm{c}, 1}, x_{\mathrm{s}, 1}, p_{\mathrm{s}, 1}\right)$ equations (15), (12) and (B2) define via $\vec{R}^{\text {out }}=S(\kappa) \vec{R}^{\text {in }}$ a symplectic linear transformation $S(\kappa)$. The contributions of $p_{\mathrm{c}, 2}^{\mathrm{in}}$ and $p_{\mathrm{s}, 2}^{\text {in }}$ to $x_{\mathrm{s}, 1}^{\text {out }}$ and $x_{\mathrm{c}, 1}^{\text {out }}$ as given in (B2) are treated as noise and do not contribute to the symplectic transformation $S$ but enter the input-output relation for the correlation matrix as an additional noise term as follows. The correlation matrix is as usually defined by $\gamma_{i, j}=\operatorname{tr}\left\{\rho\left(R_{i} R_{j}+R_{j} R_{i}\right)\right\}$. The initial state is then an $10 \times 10$ identity matrix and the final state is $\gamma^{\text {out }}=S(\kappa) S(\kappa)^{T}+\gamma_{\text {noise }}$ where the diagonal matrix 


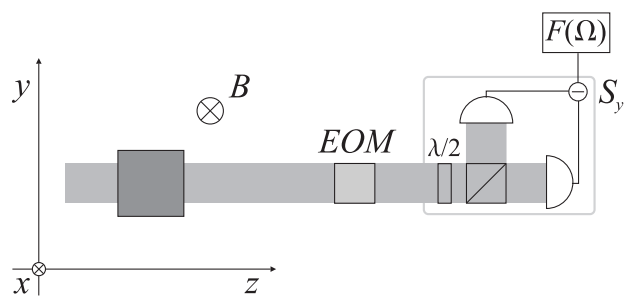

FIG. 5: Scheme for spin measurement: After the scattering a $\pi / 2$ rotation is performed on the scattered light modulated at the Larmor frequency such as to affect only the sine (cosine) component. Standard polarization measurement of $S_{y}$ and appropriate postprocessing allows to read out the mean of $X(P)$, leaving the atoms eventually in a spin squeezed state.

$\gamma_{\text {noise }}=\operatorname{diag}[0,0,0,0,0,0,1,0,1,0](\kappa / 2)^{4} / 15 \quad$ accounts for noise contributions due correlations to second order back action modes c.f. equations (B2). In order to evaluate the atomic variances after a measurement of $x_{\mathrm{c}}^{\text {out }}, p_{\mathrm{s}}^{\text {out }}, p_{\mathrm{s}, 1}^{\text {out }}$ the correlation matrix $\gamma_{\text {out }}$ is split up into blocks,

$$
\gamma_{\mathrm{out}}=\left(\begin{array}{cc}
A & C \\
C^{T} & B
\end{array}\right)
$$

where $A$ is the $2 \times 2$ subblock describing atomic variances. Now, the state $A^{\prime}$ after the measurement can be found by evaluating 32

$$
A^{\prime}=A-\lim _{x, n \rightarrow \infty} C \frac{1}{\Gamma+B} C^{T}
$$

where $\Gamma=\operatorname{diag}[1 / x, x, x, 1 / x, n, n, x, 1 / x]$ corresponds to the measured state. Note that the limit $n \rightarrow \infty$, i.e. the projection of the unobserved mode $x_{c, 2}, p_{c, 2}$ onto the identity, does not need to be taken explicitly since, remarkably, the atomic state after the measurement decouples form this mode. The conditional variances in equation (13) are then just (half the) diagonal entries of $A^{\prime}$.

\section{CONCLUSIONS}

In conclusion we have presented a simple and realistic protocol for teleportation of a coherent state, carried by a propagating pulse of light, onto the collective spin of an atomic ensemble, a suitable stationary carrier of quantum information of continuous variables. The scheme can be implemented with state of the art technique and allows to surpass any classical strategy for the transmission and storage of coherent states under realistic experimental conditions. The basic resource in the protocol is a multimode entangled state as it results form the interaction of light with atoms in the presence of an external magnetic field. We showed that Larmor precession enhances the creation of entanglement quantitatively and qualitatively. Though the interaction is not of QND type it is still possible to perform a state readout on the atomic spin as well as to create significant spin squeezing. We expect that a proper tailoring of the Larmor rotation with time dependent magnetic fields would open up interesting possibilities to further enhance the creation of entanglement and to deliberately shape scattering modes.

We acknowledge funding from the EU under project FP6-511004-COVAQIAL and support from Kompetenznetzwerk Quanteninformationsverarbeitung der Bayerischen Staatsregierung.

\section{APPENDIX A: HAMILTONIAN}

In this appendix we present a short derivation of the basic Hamiltonian (11) in order to introduce the notation used throughout this paper. More detailed descriptions can be found in 14, 15, 16, 17, 18, 19, 20, 21]. The Hamiltonian of the system is given by $H=H_{a t}+H_{l i}+V$ where the atomic part $H_{a t}=\hbar \Omega \sum_{i} F_{x}^{(i)}$ accounts for the external magnetic field along $x$ causing a ground state Zeemann splitting of $\hbar \Omega, H_{l i}$ is the free space Hamiltonian for light and the interaction term $V$ is the level shift operator [22, 33]

$$
V=\int \mathrm{d}^{3} r \vec{E}^{(-)}(\vec{r}) \overleftrightarrow{\alpha}(\vec{r}) \vec{E}^{(+)}(\vec{r})
$$

which appropriately describes the interaction of offresonant light with atoms. We assume here implicitly that the electric field contains only frequency components within a bandwidth $b$ around the carrier frequency $\omega_{c}$ of the off-resonant coherent probe pulse satisfying $\Omega \ll b \ll \Delta_{F^{\prime}}$ where the detuning $\Delta_{F^{\prime}}=\omega_{c}-\omega_{F, F^{\prime}}$.

The atomic polarizability density tensor introduced in equation (A1) is

$$
\overleftrightarrow{\alpha}(\vec{r})=\sum_{i} \overleftrightarrow{\alpha}^{(i)} \delta\left(\vec{r}-\vec{r}^{(i)}\right)
$$

where $\vec{r}^{(i)}$ is the position of atom $i$. The single atom ground state polarizability $\overleftrightarrow{\alpha}^{(i)}$ consists in general of a scalar, vector and tensor part,

$$
\overleftrightarrow{\alpha}=d^{2}\left(\alpha_{0} \mathbf{1}+\alpha_{1} \vec{F} \times+\alpha_{2} \overleftrightarrow{T}\right)
$$

where $d$ is the relevant reduced dipole matrix element of the probed transition, $\mathbf{1}$ is the $3 \times 3$ identity matrix and $\vec{F} \times$ has to be understood to give the vector cross product of $\vec{F}$ with the vector to the right. Each of the coefficients $\alpha_{j}$ is a sum of contributions from transitions to all excited states manifolds $F^{\prime}$. If the detuning is much larger than the typical excited states' hyperfine splitting, $\Delta \gg \omega_{\mathrm{hfs}}$, one finds that $\alpha_{2} \rightarrow 0$ such that the second rank polarizability can be neglected. In this case

$$
\overleftrightarrow{\alpha}=\frac{d^{2}}{\hbar(\Delta-i \Gamma / 2)}\left(a_{0} \mathbf{1}+i a_{1} \vec{F} \times\right)
$$


with real dimensionless coefficients $a_{j}$ of order unity and $\Gamma$ the excited states' decay rate. The non-hermitian part of the resulting Hamilton operator describes the effect of light absorption and loss of ground state population due to depumping in the course of interaction. In the following we will focus on the coherent interaction and, for the time being, take into account only the hermitian component. The effects of light absorption and atomic depumping are treated below.

Coherent interaction Since scattering of light occurs predominantly in the forward direction [22] it is legitimate to adopt a one dimensional model such that the (negative frequency component of the) electric field propagating along $z$ is given by

$$
\begin{aligned}
\vec{E}^{(-)}(z, t) & =E^{(-)}(z) \vec{e}_{y}+\mathcal{E}^{(-)}(z, t) \vec{e}_{x} \\
E^{(-)}(z) & =\rho\left(\omega_{c}\right) \int_{b} \mathrm{~d} \omega a^{\dagger}(\omega) e^{-i k z} \\
\mathcal{E}^{(-)}(z, t) & =\rho\left(\omega_{c}\right) \sqrt{4 \pi N_{p h} / T} e^{-i\left(k_{c} z-\omega_{c} t\right)}
\end{aligned}
$$

where $\rho(\omega)=\sqrt{\hbar \omega_{c} / 4 \pi \epsilon_{0} A c}$ and $A$ denotes the pulse's cross sectional area, $N_{p h}$ the overall number of photons in the pulse and $T$ its duration. We restrict the field in $x$ polarization to the classical probe pulse, since only the coupling of atoms to the $y$ polarization is enhanced by the coherent probe. Furthermore we implicitly assume for the classical pulse a slowly varying envelope such that it arrives at $z=0$ at $t=0$ and is then constant for a time $T$. Combining this expression for the field with expressions (A2) and (A3) for the atomic polarizability in equation (A1) yields

$V=-\frac{i \hbar \kappa}{\sqrt{4 \pi J T}} \int_{b} \mathrm{~d} \omega \int \mathrm{d} z j(z)\left(a(\omega) e^{-i\left[\left(k_{c}-k\right) z-\omega_{c} t\right]}-\right.$ h.c. $)$

where we defined a dimensionless coupling constant $\kappa=\sqrt{N_{p h} J} \omega_{c} a_{1} d^{2} / \hbar \epsilon_{0} c A \Delta$ and an atomic spin density $j_{z}(z)=\sum_{i} F_{z}^{(i)} \delta\left(z-z^{(i)}\right)$. In this expression we skipped terms proportional to $a_{0}$ which will give rise only to a global phase shift and included for convenience the square root factor with $J=N_{a t} F$ where $N_{a t}$ is the number of atoms. Note that the coupling can be expressed as $\kappa=\sqrt{N_{p h} J} a_{1} \sigma \Gamma / 2 A \Delta$ with $\sigma$ the scattering cross section on resonance.

We now define field quadratures for spatially localized modes [24, 25] as

$$
\begin{aligned}
& x(z)=\frac{1}{\sqrt{4 \pi}} \int_{b} \mathrm{~d} \omega\left(a(\omega) e^{-i\left(k_{c}-k\right) z}+\text { h.c. }\right),(\mathrm{A} 4 \mathrm{a}) \\
& p(z)=-\frac{i}{\sqrt{4 \pi}} \int_{b} \mathrm{~d} \omega\left(a(\omega) e^{-i\left(k_{c}-k\right) z}-\text { h.c. }\right)(\mathrm{A} 4 \mathrm{~b})
\end{aligned}
$$

with commutation relations $\left[x(z), p\left(z^{\prime}\right)\right]=i c \delta\left(z-z^{\prime}\right)$ where the delta function has to be understood to have a width on the order of $c / b$. Since we assumed that $\Omega \ll b$, the time it takes for such a fraction of the pulse to cross the ensemble is much smaller than the Larmor period
$1 / \Omega$. During the interaction with one of these spatially localized modes the atomic state does not change appreciable and we can simplify the interaction operator to $V=\hbar \kappa(J T)^{-1 / 2} J_{z} p(0)$ where $J_{z}=\sum_{i} F_{z}^{(i)}$ and we assumed that the ensemble is located at $z=0$ and changed to a frame rotating at the carrier frequency $\omega_{c}$.

A last approximation concerns the description of the atomic spin state. Initially the sample is prepared in a coherent spin state with maximal polarization along $x$, i.e. in the eigenstate of $J_{x}$ with maximal eigenvalue $J$. We can thus make use of the Holstein-Primakoff approximation [23] which allows to describe the spin state as a Gaussian state of a single harmonic oscillator. The first step is to express collective step up/down operators (along $x$ ), $J_{ \pm}=J_{y} \pm i J_{z}$, in terms of bosonic creation and annihilation operators, $\left[b, b^{\dagger}\right]=\mathbb{1}$, as

$$
J_{+}=\sqrt{2 J} \sqrt{\mathbb{1}-b^{\dagger} b / 2 J} b, \quad J_{-}=\sqrt{2 J} b^{\dagger} \sqrt{\mathbb{1}-b^{\dagger} b / 2 J} .
$$

It is easily checked that these operators satisfy the correct commutation relations $\left[J_{+}, J_{-}\right]=2 J_{x}$ if one identifies $J_{x}=J-b^{\dagger} b$. The fully polarized initial state thus corresponds to the ground state of the harmonic oscillator. Note that this mapping is exact. Under the condition that $\left\langle b^{\dagger} b\right\rangle \ll J$ one can approximate $J_{+} \simeq \sqrt{2 J} b, J_{-} \simeq \sqrt{2 J} b^{\dagger}$ and therefore $J_{z} \simeq-i \sqrt{J / 2}\left(b-b^{\dagger}\right)$. Introducing atomic quadratures $X=\left(b+b^{\dagger}\right) / \sqrt{2}$ and $P=-i\left(b-b^{\dagger}\right) / \sqrt{2}$ finally yields the desired expression for the interaction $V=\hbar \kappa T^{-1 / 2} P p(0)$.

In terms of atomic quadratures the free Hamiltonian for atoms is $H=\hbar \Omega / 2\left(X^{2}+P^{2}\right)$. In the frame rotating at the carrier frequency the action of $H_{l i}$ on the light quadratures $x(z), p(z)$ is simply $i / \hbar\left[H_{l i}, x(z)\right]=-c \partial_{z} x(z)$ and likewise for $p(z)$.

Noise effects The antihermitian part of the level shift operator (A1) describes depumping of ground state population and photon absorption. The effect of the latter process can - as far as it concerns the performance of the teleportation protocol - be treated on equal footing with mode mismatch and finite detector efficiency. This is done in section ulation on the other hand will eventually cause degrading of atomic polarization due to spontaneous emission events. For a single atom the dominating term describing this process stems from the scalar part of the polarizability and is given by $V_{\text {loss }}=i \hbar \eta \mathbb{1} / 4 T$ where $\eta=N_{p h} a_{0} \omega_{c} \Gamma d^{2} / 2 \hbar \Delta^{2} \epsilon_{0} A c=N_{p h} a_{0} \sigma \Gamma^{2} / 4 A \Delta^{2}$. It is possible to have $\eta \ll 1$ and at the same time a large coupling $\kappa \simeq 1$. For a thermal cloud of atoms an additional source of decoherence are light assisted collisions which in fact dominate the decay process. Assuming a transverse relaxation at an overall rate $\beta / T$ with $\beta \precsim .2$ the exponential decay during the interaction can to a good approximation be treated linearly which leads to equations (10). The Langevin noise operators $f_{X, P}$ can in principle be derived by a microscopic model as is done in 17] for dephasing due spontaneous emission. 


\section{APPENDIX B: BACK ACTION AND INPUT MODES}

Back action We evaluate here the input/output relations (5c5d) for the cosine and sine components of the in-phase field. For the former we take equation (3d) at $\xi=c \tau, t=T$, multiply by $\sqrt{2 / T} \cos (\Omega \tau)$ and integrate over $\tau$ from 0 to $T$. Using equations (3a 3b and the approximate orthogonality of $\cos (\Omega \tau)$ and $\sin (\Omega \tau)$ one finds

$$
\begin{aligned}
x_{\mathrm{c}}^{\text {out }}= & x_{\mathrm{c}}^{\text {in }}+\frac{\kappa}{\sqrt{2}} P^{\text {in }}+ \\
& +\frac{\sqrt{2} \kappa^{2}}{T^{3 / 2}} \int_{0}^{T} \mathrm{~d} \tau \int_{0}^{\tau} \mathrm{d} \tau^{\prime}\left[\cos (\Omega \tau)^{2} \sin \left(\Omega \tau^{\prime}\right) \bar{p}\left(c \tau^{\prime}, 0\right)-\right. \\
& \left.-\cos (\Omega \tau) \sin (\Omega \tau) \cos \left(\Omega \tau^{\prime}\right) \bar{p}\left(c \tau^{\prime}, 0\right)\right] .
\end{aligned}
$$

After interchanging the order of integration, $\int_{0}^{T} \mathrm{~d} \tau \int_{0}^{\tau} \mathrm{d} \tau^{\prime} \rightarrow \int_{0}^{T} \mathrm{~d} \tau^{\prime} \int_{\tau^{\prime}}^{T} \mathrm{~d} \tau$ one can perform the integration over $\tau$. Neglecting all terms of order $n_{0}^{-1}$ or less where $n_{0}=\Omega T \gg 1$ one finds

$x_{\mathrm{c}}^{\text {out }}=x_{\mathrm{c}}^{\text {in }}+\frac{\kappa}{\sqrt{2}} P^{\text {in }}+\frac{\sqrt{2} \kappa^{2}}{T^{3 / 2}} \int_{0}^{T} \mathrm{~d} \tau \frac{T-\tau}{2} \sin (\Omega \tau) \bar{p}(c \tau, 0)$.

The last term represents back action of light onto itself. It can be expressed as a sum of two terms, one proportional to $p_{\mathrm{s}}^{\text {in }}$ and another one proportional to

$$
p_{\mathrm{s}, 1}^{\mathrm{in}}=\sqrt{3}\left(\frac{2}{T}\right)^{3 / 2} \int_{0}^{T} \mathrm{~d} \tau\left(\frac{T}{2}-\tau\right) \sin (\Omega \tau) \bar{p}(c \tau, 0) .
$$

It is easily verified that the back action mode defined by this equation and the corresponding expression for $x_{\mathrm{s}}^{\text {back }}$ is canonical $\left[x_{\mathrm{s}, 1}^{\mathrm{in}}, p_{\mathrm{s}, 1}^{\mathrm{in}}\right]=i\left[1-\mathcal{O}\left(n_{0}^{-2}\right)\right] \simeq i$ and independent from all the other modes introduced so far, f.e. $\left[x_{\mathrm{s}}^{\text {in }}, p_{\mathrm{s}, 1}^{\text {in }}\right]=\mathcal{O}\left(n_{0}^{-2}\right) \simeq 0$. The variance is thus $\left(\Delta p_{\mathrm{s}, 1}^{\text {in }}\right)^{2}=1 / 2$. Repeating the calculation for $x_{\mathrm{s}}^{\text {out }}$ with appropriate replacements and a definition of $p_{\mathrm{c}, 1}^{\text {in }}$ analogous to equation (B1) finally yields equations $(5 \mathrm{5c} 5 \mathrm{dd})$.

In a similar way input-output relations for the back action modes itself are derived. In particular for the in phase components one finds

$$
\begin{aligned}
& x_{\mathrm{c}, 1}^{\text {out }}=x_{\mathrm{c}, 1}^{\text {in }}-\frac{1}{\sqrt{3}}\left(\frac{\kappa}{2}\right)^{2} p_{\mathrm{s}}^{\text {in }}+\frac{1}{\sqrt{15}}\left(\frac{\kappa}{2}\right)^{2} p_{\mathrm{s}, 2}^{\text {in }}, \\
& x_{\mathrm{s}, 1}^{\text {out }}=x_{\mathrm{s}, 1}^{\text {in }}-\frac{1}{\sqrt{3}}\left(\frac{\kappa}{2}\right)^{2} p_{\mathrm{c}}^{\text {in }}+\frac{1}{\sqrt{15}}\left(\frac{\kappa}{2}\right)^{2} p_{\mathrm{c}, 2}^{\text {in }} .
\end{aligned}
$$

In both equations the third terms on the right hand side describe contributions of second order back action modes defined by

$$
p_{\mathrm{s}, 2}^{\text {in }}=6\left(\frac{10}{T^{5}}\right)^{1 / 2} \int_{0}^{T} \mathrm{~d} \tau\left(\frac{T^{2}}{6}-T \tau+\tau^{2}\right) \sin (\Omega \tau) \bar{p}(c \tau, 0)
$$

and similarly for $x_{\mathrm{s}, 2}^{\mathrm{in}}$ and the cosine component. These modes are again canonical and independent. As a sidemark we note that, formally, it is possible to define scattering modes of arbitrary order whose mode functions are given in general by products of Legendre polynomials and $\cos (\Omega t)[\sin (\Omega t)]$ resulting in a hierarchy of input-output relations similar to (B2).

Input state The input field, propagating along the positive $y$ direction and polarized along $z$ (see figure 2), is described by operators $\left[b(\omega), b^{\dagger}\left(\omega^{\prime}\right)\right]=\delta\left(\omega-\omega^{\prime}\right)$ in frequency space and $\left[\hat{y}(y), \hat{q}\left(y^{\prime}\right)\right]=i c \delta\left(y-y^{\prime}\right)$ in real space. ( $\hat{y}$ is the quadrature operator for the field in-phase and $y$ on the other hand is the position along the $y$-direction.) In analogy to equation (A4) these bases are connected via

$$
\begin{aligned}
& \hat{y}(y)=\frac{1}{\sqrt{4 \pi}} \int_{b} \mathrm{~d} \omega\left(b(\omega) e^{-i\left(k_{c}-k\right) y}+\text { h.c. }\right), \\
& \hat{q}(y)=-\frac{i}{\sqrt{4 \pi}} \int_{b} \mathrm{~d} \omega\left(b(\omega) e^{-i\left(k_{c}-k\right) y}-\text { h.c. }\right) .
\end{aligned}
$$

As shown in section $\llbracket A$ we can teleport the mode

$$
y=\frac{1}{\sqrt{2}}\left(y_{\sin }+q_{\cos }\right), \quad q=-\frac{1}{\sqrt{2}}\left(y_{\cos }-q_{\sin }\right)
$$

and the claim is that this corresponds to a pulse at the upper sideband $\omega_{c}+\Omega$. Using the definitions of cosine and sine modes (44) as well as $\hat{\bar{y}}(c \tau, 0)=\hat{y}(-c \tau, 0)$ and the same for $\hat{q}(y)$ we have

$$
\begin{aligned}
y & =\frac{1}{\sqrt{T}} \int_{0}^{T} \mathrm{~d} \tau[\sin (\Omega \tau) \hat{\bar{y}}(c \tau, o)+\cos (\Omega \tau) \hat{\bar{q}}(c \tau, 0)] \\
& =\frac{-i}{\sqrt{4 \pi T}} \int_{0}^{T} \mathrm{~d} \tau \int_{b} \mathrm{~d} \omega\left[b(\omega) e^{i\left(\omega_{c}+\Omega-\omega\right) \tau}-h . c .\right] \\
q & =-\frac{1}{\sqrt{T}} \int_{0}^{T} \mathrm{~d} \tau[\cos (\Omega \tau) \hat{\bar{y}}(c \tau, o)-\sin (\Omega \tau) \hat{\bar{q}}(c \tau, 0)] \\
& =\frac{-1}{\sqrt{4 \pi T}} \int_{0}^{T} \mathrm{~d} \tau \int_{b} \mathrm{~d} \omega\left[b(\omega) e^{i\left(\omega_{c}+\Omega-\omega\right) \tau}+h . c .\right]
\end{aligned}
$$

To explicitly see that this corresponds to a pulse centered at the upper sideband it is convenient to change to a more precise model by replacing the $1 / \sqrt{T}$ factor, which is just the pulse's slowly varying amplitude function in a simple square well approximation, by a function $A(\tau)$ of dimension $s^{-1 / 2}$ normalized such that $\int_{0}^{T} \mathrm{~d} \tau|A(\tau)|^{2}=$ 1. Its Fourier transform $A(\omega)=\frac{1}{\sqrt{2 \pi}} \int_{0}^{T} \mathrm{~d} \tau A(\tau) \exp (i \omega \tau)$ is centered at zero and has a width $1 / T=\Delta \omega \ll \Omega$ in accord with our condition $1 \ll \Omega T$. Replacing now $1 / \sqrt{T}$ by $A(\tau)$ (of course inside the integral over $\tau$ ) in the expressions for $y$ and $q$ yields

$$
\begin{aligned}
y & =\frac{-i}{\sqrt{4 \pi}} \int_{0}^{T} \mathrm{~d} \tau A(\tau) \int_{b} \mathrm{~d} \omega\left[b(\omega) e^{i\left(\omega_{c}+\Omega-\omega\right) \tau}-h . c .\right] \\
& =\frac{-i}{\sqrt{2}} \int_{b} \mathrm{~d} \omega\left[A\left(\omega_{c}+\Omega-\omega\right) b(\omega)-h . c .\right], \\
q & =\frac{-1}{\sqrt{2}} \int_{b} \mathrm{~d} \omega\left[A\left(\omega_{c}+\Omega-\omega\right) b(\omega)+h . c .\right] .
\end{aligned}
$$

This is evidently a mode whose spectral mode function is the same as the classical pulse but is centered at $\omega_{c}+\Omega$. 


\section{APPENDIX C: FEEDBACK}

The feedback in continuous variable quantum teleportation is sometimes described by equations equivalent to (8) but with a classical random variable describing the measurement outcome in place of the operators corresponding to the chosen displacement, which - though giving the right result - is mathematically questionable. We point out that relations (8) hold stricto sensu as operator identities. This is true for mixed and even for non-gaussian states, as we will show below.

Consider a bipartite system of $N+1$ modes and denote the first mode as system $A$ and the remaining $N$ modes as system $B$. Let the state of the compound system be given by $\rho_{A B}$. Our aim here is to describe protocols which consist of the following steps:

Measurement On system $B$ a set of commuting observables $\left\{\hat{r}_{1}, \ldots, \hat{r}_{N}\right\}$ is measured where each of the operators $\hat{r}_{i}$ is either $x_{i}$ or $p_{i}$, one of the quadratures of mode $i$ in $B$. Let the corresponding measurement outcomes $r_{i}$ be arranged in a vector $\vec{R}^{B}=\left(r_{1}, \ldots, r_{N}\right)$. With the eigenvalue equation $\hat{r}_{i}\left|r_{i}\right\rangle_{B}=r_{i}\left|r_{i}\right\rangle_{B}$, where $\left|r_{i}\right\rangle_{B}$ is the generalized eigenstate of $\hat{r}_{i}$, the normalized state of system $A$ conditioned on the measurement outcomes is

$$
\rho_{A}^{(1)}(\vec{R})={ }_{B}\left\langle r_{1}, \ldots, r_{N}\left|\rho_{A B}\right| r_{1}, \ldots, r_{N}\right\rangle_{B} / p(\vec{R}) .
$$

$p(\vec{R})$ is the probability to get the measurement outcomes $\vec{R}$ and is normalized as $\int \mathrm{d}^{N} r p(\vec{R})=1$.

Feedback Depending on the measurement outcomes system $A$ is then displaced in $x_{A}$ and $p_{A}$ by an amount $\vec{R} \vec{g}_{x}^{T}$ and $\vec{R} \vec{g}_{p}^{T}$ respectively where $\vec{g}_{x(p)}$ are any real $N$ dimensional (row) vectors determining the strength with which each outcome is fed back into system $A$. In teleportation protocols these coefficients are usually referred to as gains. The state of system $A$ after the feedback operation is then

$$
\rho_{A}^{(2)}(\vec{R})=D_{A}^{\dagger} \rho_{A}^{(1)}(\vec{R}) D_{A} .
$$

$D_{A}=D_{A}\left(\vec{R} \vec{g}_{x}^{T}, \vec{R} \vec{g}_{p}^{T}\right) \doteq \exp \left(i \vec{R} \vec{g}_{x}^{T} p_{A}\right) \exp \left(-i \vec{R} \vec{g}_{p}^{T} x_{A}\right) \quad$ is the unitary displacement operator effecting the desired transformations $D_{A} x_{A} D_{A}^{\dagger}=x_{A}+\vec{R} \vec{g}_{x}^{T}$ and $D_{A} p_{A} D_{A}^{\dagger}=p_{A}+\vec{R} \vec{g}_{p}^{T}$.

Ensemble average On average over all measurement outcomes, weighted with their respective probabilities, the state of system $A$ is

$$
\bar{\rho}_{A}=\int \mathrm{d}^{N} r p(\vec{R}) \rho_{A}^{(2)}(\vec{R}) .
$$

Combining this with the expressions for $\rho_{A}^{(2)}$ and $\rho_{A}^{(1)}$ above one can express

$$
\begin{aligned}
\bar{\rho}_{A} & =\int \mathrm{d}^{N} r D_{A B}^{\dagger}\left\langle r_{1}, \ldots, r_{N}\left|\rho_{A B}\right| r_{1}, \ldots, r_{N}\right\rangle_{B} D_{A} \\
& =\int \mathrm{d}^{N} r_{B}\left\langle r_{1}, \ldots, r_{N}\left|D_{A B}^{\dagger} \rho_{A B} D_{A B}\right| r_{1}, \ldots, r_{N}\right\rangle_{B} \\
& =\operatorname{tr}_{B}\left\{D_{A B}^{\dagger} \rho_{A B} D_{A B}\right\}
\end{aligned}
$$

where the trace in the last line is now taken with respect to both system $A$ and $B$. In going from the first line to the second line we made use of the identity

$$
\left|r_{1}, \ldots, r_{N}\right\rangle_{B} D_{A}=D_{A B}\left|r_{1}, \ldots, r_{N}\right\rangle_{B}
$$

with the unitary operator $D_{A B}$ defined as $D_{A B}=$ $D_{A B}\left(\hat{\vec{R}} \vec{g}_{x}^{T}, \hat{\vec{R}} \vec{g}_{p}^{T}\right) \doteq \exp \left(i \hat{\vec{R}} \vec{g}_{x}^{T} p_{A}\right) \exp \left(-i \hat{\vec{R}} \vec{g}_{p}^{T} p_{A}\right)$ where $\hat{\vec{R}}=\left(\hat{r}_{1}, \ldots, \hat{r}_{N}\right)$ is now the vector of operators $\hat{r}_{i}^{B}$ and $D_{A B}$ acts on both systems $A$ and $B$. Note that identity (C2) is valid only for commuting observables $\hat{r}_{i}^{B}$. The resulting equation (C1) is the key point in this consideration.

Observables in the ensemble average Consider finally the mean of, for example, $x_{A}$ after the measurement and feedback procedure, i.e. with respect to the ensemble averaged state $\bar{\rho}_{A}$. It is given by

$$
\begin{aligned}
\left\langle x_{A}\right\rangle & =\operatorname{tr}_{A}\left\{x_{A} \bar{\rho}_{A}\right\}=\operatorname{tr}_{A B}\left\{D_{A B} x_{A} D_{A B}^{\dagger} \rho_{A B}\right\} \\
& =\operatorname{tr}_{A B}\left\{\left(x_{A}+\hat{\vec{R}} \vec{g}_{x}^{T}\right) \rho_{A B}\right\} .
\end{aligned}
$$

From this identity and the corresponding expression for $\left\langle p_{A}\right\rangle$, which both are true for all initial states $\rho_{A B}$, we can deduce the operator identities

$$
x_{A}^{\mathrm{fin}}=x_{A}+\hat{\vec{R}} \vec{g}_{x}^{T}, \quad p_{A}^{\mathrm{fin}}=p_{A}+\hat{\vec{R}} \vec{g}_{p}^{T}
$$

where $x_{A}^{\mathrm{fin}}, p_{A}^{\mathrm{fin}}$ describe the final state of system $A$ in the Heisenberg picture and means have to be taken with respect to the unchanged initial state of both systems $A$ and $B$. If $\rho_{A B}$ is a pure Gaussian state the last two equations fully determine the final state $\bar{\rho}_{A}$. This was used in equation (8). Note that these considerations are easily extended to situations in which system $A$ consists of more than one mode.
[1] D. Bouwmeester et al., Nature 390, 575 (1997), D. Fattal et al., Phys. Rev. Lett. 92, 037904 (2004), I. Marcikic et al., Nature 421, 509 (2003), Y.-H. Kim et al., Phys. Rev.
Lett. 86, 1370 (2001), D. Boschi et al., Phys. Rev. Lett. 80, 1121 (1998), R. Ursinn et al., Nature 430, 849 (2004), J.-W. Pan et al., Phys. Rev. Lett. 86, 4435 (2001), A. 
Furusawa et al., Science 282, 706 (1998), T.C. Zhang et al., Phys. Rev. A 67, 033802 (2003), W.P. Bowen et al., Phys. Rev. A 67, 032302 (2003), H. Yonezawa et al., Nature 431, 430 (2004), N. Takei et al., Phys. Rev. Lett. 94220502 (2005)

[2] M.Riebe et al., Nature 429, 734 (2004), M.D. Barrett et al., Nature 429, 737 (2004)

[3] B. Julsgaard, J. Sherson, J.I. Cirac, J. Fiurasek, E.S. Polzik, Nature 432, 482 (2004)

[4] S. Braunstein and A. Pati, Quantum Information with Continuous Variables, Kluwer, New York, 2003

[5] M.J. Holland, M.J. Collett, D.F. Walls, M.D. Levenson, Phys. Rev. A 42, 2995 (1990)

[6] J.P. Poizat, J.F. Roch, P. Grangier, Ann. Phys. Fr. 19, 265 (1994)

[7] B. Julsgaard, A. Kozhekin, E. S. Polzik, Nature 413, 400 (2001)

[8] L. Vaidman, Phys. Rev. A, 49, 1473 (1994)

[9] S.L. Braunstein, H.J. Kimble, Phys. Rev. Lett. 80, 869 (1998)

[10] A. Kuzmich, L. Mandel, N.P. Bigelow, Phys. Rev. Lett. 85, 1594 (2000)

[11] J.M. Geremia, J.K. Stockton, H. Mabuchi, Science 304, $270(2004)$

[12] T. Opatrny, J. Fiurasek, Phys. Rev. Lett. 95, 053602 (2005)

[13] L. Mista, R. Filip, Phys. Rev. A 71032342 (2005)

[14] A. Kuzmich, K. Mølmer, E.S. Polzik, Phys. Rev. Lett. 79, 4782 (1997), E.S. Polzik, Phys. Rev. A 59, 4202 (1999)

[15] A. Kuzmich, N.P. Bigelow, L. Mandel, Europhys. Lett. 42, 481 (1998)

[16] L.K. Thomsen, S. Mancini, H.M. Wiseman, Phys. Rev. A 65, 061801 (2002)

[17] L.-M. Duan, J.I. Cirac, P. Zoller, E.S. Polzik, Phys. Rev.
Lett. 85, 5643 (2000)

[18] A. E. Kozhekin, K. Mølmer, E.S. Polzik, Phys. Rev. A 62, 033809 (2000)

[19] B. Julsgaard, C. Schori, J.L. Sørensen, E.S. Polzik., Quant. Inform. and Comp., spec. issue 3, 518 (2003)

[20] B. Julsgaard, Entanglement and Quantum Interactions with Macroscopic Gas Samples, $\mathrm{PhD}$ Thesis, October 2003, Aarhus University, Denmark, http://www.nbi.dk/ julsgard/

[21] J. Sherson, B. Julsgaard, E.S. Polzik, quant-ph/0408146

[22] D.V. Kupriyanov, O.S. Mishina, I.M. Sokolov, B. Julsgaard, E.S. Polzik, quant-ph/0411083

[23] C. Kittel, Quantum Theory of Solids, Wiley, New York, 1987

[24] A. Silberfarb, I.H. Deutsch, Phys. Rev. A 68, 13817 (2003)

[25] L.B. Madsen, K. Mølmer, Phys. Rev. A 70, 052324 (2004)

[26] C.M. Caves, B.L. Schumaker, Phys. Rev. A 31, 3068 (1985), B.L. Schumaker, C.M. Caves, Phys. Rev. A 31, 3093 (1985)

[27] G. Giedke, B. Kraus, M. Lewenstein, J.I. Cirac, Phys. Rev. A 64, 052303 (2001)

[28] S.L. Braunstein, H.J. Kimble, C.A. Fuchs, J. Mod. Opt. 47, 267 (2000)

[29] K. Hammerer, M.M. Wolf, E.S. Polzik, J.I. Cirac, Phys. Rev. Lett. 94, 150503 (2005)

[30] G. Giedke, M. M. Wolf, O. Krger, R. F. Werner, J. I. Cirac Phys. Rev. Lett. 91, 107901 (2003)

[31] P. Grangier, J.A. Levenson, J.P. Poizat, Nature 396, 537 (1998)

[32] G. Giedke, J.I. Cirac, Phys. Rev. A 66, 032316 (2002)

[33] W. Happer, Rev. Mod. Phys. 44, 169 (1972) 